\title{
Quark and lepton flavor model with leptoquarks in a modular $A_{4}$ symmetry
}

\author{
Takaaki Nomura ${ }^{1, \mathrm{a}}$, Hiroshi Okada ${ }^{2,3, \mathrm{~b}}$, Yuta Orikasa $^{4, \mathrm{c}}$ (1) \\ ${ }^{1}$ College of Physics, Sichuan University, Chengdu 610065, China \\ 2 Asia Pacific Center for Theoretical Physics, Pohang 37673, Republic of Korea \\ ${ }^{3}$ Department of Physics, Pohang University of Science and Technology, Pohang 37673, Republic of Korea \\ ${ }^{4}$ Institute of Experimental and Applied Physics, Czech Technical University in Prague, 11000 Prague 1, Czech Republic
}

Received: 8 July 2021 / Accepted: 16 September 2021 / Published online: 25 October 2021

(C) The Author(s) 2021

\begin{abstract}
We propose a quark-lepton model via leptoquarks and modular $A_{4}$ symmetry. Since the neutrino mass is induced at one-loop level mediated by down quarks as well as leptoquarks, we have to explain lepton and quark masses and mixings with a single modulus $\tau$. Here, we find predictions for lepton and quark sectors with unified modulus $\tau$, and show several constraints originating from leptoquarks.
\end{abstract}

\section{Introduction}

Since lepto-quark(LQ) bosons connect lepton and quark sectors, these models potentially explain several new physics beyond the standard model (SM); e.g., lepton(muon or electron) anomalous magnetic dipole moment $(g-2)$ [1-6], $B$ meson decays such as $b \rightarrow s \mu \bar{\mu}[2,4,5,7-9]$ and $b \rightarrow$ $c \ell \bar{v}_{\ell}(\ell=e, \mu, \tau)[4,8-10],{ }^{1}$ and nonzero neutrino masses $[6,9,11,14]$. Especially, muon $g-2$ anomaly is recently reported by E989 experiment at Fermilab combining BNL result [22], and its value is deviated from the SM by $4.2 \sigma$ as follows:

$\Delta a_{\mu}=(25.1 \pm 5.9) \times 10^{-10}$.

Also, the LHCb collaboration [23] recently reported anomaly of rare $B$ meson decays of $b \rightarrow s \mu \bar{\mu}$ that is understood as

\footnotetext{
$\overline{{ }^{1} \text { The anomaly }}$ of $b \rightarrow c \ell \bar{v}_{\ell}$ processes are observed in experiments [15-21], and LQ model is one of the most promising explanations on this anomaly.

a e-mail: nomura@scu.edu.cn

b e-mail: hiroshi.okada@apctp.org

c e-mail: Yuta.Orikasa@utef.cvut.cz (corresponding author)
}

violation of lepton universality. The updated result is given by

$$
\begin{aligned}
& \frac{B R\left(B^{+} \rightarrow K^{+} \mu^{-} \mu^{+}\right)}{B R\left(B^{+} \rightarrow K^{+} e^{-} e^{+}\right)} \\
& \quad=0.846_{-0.039-0.012}^{+0.042+0.013} \quad\left(1.1 \mathrm{GeV}^{2}<q^{2}<6 \mathrm{GeV}^{2}\right)
\end{aligned}
$$

where first(second) uncertainty is statistical(systematic) one and $q^{2}$ is the invariant mass squared for dilepton. In addition to the above phenomenologies, interestingly, the nonzero Majorana neutrino mass at one-loop can be realized without any additional symmetries by introducing appropriate LQs [12-14]. This may be natural realization of tiny neutrino mass model due to loop suppression.

Considering above issues, one finds that Yukawa flavor structure is also very important to explain them. Recently, powerful symmetries to restrict the number of parameters in Yukawa couplings, so called "modular flavor symmetries", were proposed by authors in Refs. [24,25], in which they have applied modular originated non-Abelian discrete flavor symmetries to quark and lepton sectors. One remarkable advantage of applying this symmetries is that dimensionless couplings of model can be transformed into non-trivial representations under those symmetries, and all the dimensionless values are uniquely fixed once modulus is determined in fundamental region. We then do not need the scalar fields to obtain a predictive mass matrix. Along the line of this idea, a vast reference has recently appeared in the literature, e.g., $A_{4}$ [24,26-58], $S_{3}$ [59-64], $S_{4}$ [65-76], $A_{5}$ [70,77,78], double covering of $A_{5}$ [79-81], larger groups [82], multiple modular symmetries [83], and double covering of $A_{4}$ [84,85], $S_{4}$ [86,87], and the other types of groups [8893] in which masses, mixing, and CP phases for the quark 
and/or lepton have been predicted. ${ }^{2}$ Moreover, a systematic approach to understand the origin of CP transformations has been discussed in Ref. [102], and CP/flavor violation in models with modular symmetry was discussed in Refs. [56,103105], and a possible correction from Kähler potential was discussed in Ref. [106]. Furthermore, systematic analysis of the fixed points (stabilizers) has been discussed in Ref. [107]. A very recent paper of Ref. [108] finds a favorable fixed point $\tau=\omega$ among three fixed points, which are the fundamental domain of PSL $(2, Z)$, by systematically analyzing the stabilized moduli values in the possible configurations of flux compactifications as well as investigating the probabilities of moduli values. It is then interesting to discuss a LQ model under the framework of modular flavor symmetry since we are motivated to consider lepton and quark sector together as a LQ connect these sectors and some predictions in both sector can be expected.

In this paper, we focus on the quark and lepton masses and mixings based on a LQ model in Ref. [14], introducing modular $A_{4}$ symmetry to reduce free parameters of Yukawa couplings. Since the quark sector connects to the lepton sector via LQ, charge assignments for quarks(leptons) directly affect the leptons(quarks). In this sense, it would be a good motivation towards unification of quark and lepton flavor in $A_{4}$ modular symmetry.

This paper is organized as follows. In Sect. 2, we review our model of quark and lepton. In Sect. 3, we have numerical analysis and show several results for normal and inverted hierarchies. We conclude in Sect. 4. In appendix, we summarize several features of modular $A_{4}$ symmetry.

\section{Model}

In this section, we review our model. It is known that introducing proper leptoquarks lead us to a radiative seesaw model without any additional symmetries such as $Z_{2}$. Here, we introduce two types of leptoquarks $\eta$ and $\Delta$ based on Ref. [14]. The color-triplet $\eta$ has $S U(2)_{L}$ doublet with $1 / 6$ hypercharge, and the color-antitriplet $\Delta$ has $S U(2)_{L}$ triplet with $1 / 3$ hypercharge, where these new bosons and their charges are summarized in Table 1. Then, the valid Lagrangian to induce the quark and lepton mass matrices is given by

$-\mathcal{L}_{Y}^{q}=y_{i j}^{u} \bar{u}_{R_{i}}\left(i \sigma_{2}\right) H^{*} Q_{L_{j}}+y_{i j}^{d} \bar{d}_{R_{i}} H Q_{L_{j}}+$ h.c.,

$-\mathcal{L}_{Y}^{\ell}=h_{i j} \bar{e}_{R_{i}} H L_{L_{j}}+$ h.c..

\footnotetext{
${ }^{2}$ For interest readers, we provide some literature reviews, which are useful to understand the non-Abelian group and its applications to flavor structure [94-101].
}

The Lagrangian for the mixing between the quark and lepton and nontrivial potential are given by

$$
-\mathcal{L}_{Y}^{m i x}=f_{i j} \overline{d_{R_{i}}} \eta^{T}\left(i \sigma_{2}\right) L_{L_{j}}+g_{i j} \overline{Q_{L_{i}}^{c}}\left(i \sigma_{2}\right) \Delta L_{L_{j}}+\text { h.c. },
$$

$\mathcal{V} \supset-\mu H^{\dagger} \Delta \eta+$ h.c.

where $(i, j)=1-3$ are family indices, $\sigma_{2}$ is the second Pauli matrix, and $H$ is the SM Higgs field that develops a nonzero VEV, which is symbolized by $\langle H\rangle \equiv v / \sqrt{2} \approx 246 / \sqrt{2} \mathrm{GeV}$, and $H$ has nonzero modular weight. Here, we parameterize components of the scalars as follows:

$H=\left[\begin{array}{c}w^{+} \\ \frac{v+\phi+i z}{\sqrt{2}}\end{array}\right], \quad \eta=\left[\begin{array}{c}\eta_{2 / 3} \\ \eta_{-1 / 3}\end{array}\right], \quad \Delta=\left[\begin{array}{cc}\frac{\delta_{1 / 3}}{\sqrt{2}} & \delta_{4 / 3} \\ \delta_{-2 / 3} & -\frac{\delta_{1 / 3}}{\sqrt{2}}\end{array}\right]$,

where the subscript of the fields represents the electric charge, and $w^{+}$and $z$ are absorbed by the longitudinal component of the $W^{+}$and $Z$ bosons, respectively. Due to the $\mu$ term in Eq. (2.4), the charged components with $1 / 3$ and $2 / 3$ electric charges mix each other. Here, we parametrize their mixing matrices and mass eigenstates as follows:

$$
\left[\begin{array}{c}
\eta_{i / 3} \\
\delta_{i / 3}
\end{array}\right]=O_{i}\left[\begin{array}{c}
A_{i} \\
B_{i}
\end{array}\right], \quad O_{i} \equiv\left[\begin{array}{cc}
c_{a_{i}} & s_{a_{i}} \\
-s_{a_{i}} & c_{a_{i}}
\end{array}\right], \quad(i=1,2),
$$

where their masses are denoted as $m_{A_{i}}$ and $m_{B_{i}}$ respectively. The interactions in terms of the mass eigenstates can be written as

$$
\begin{aligned}
& -\mathcal{L}_{Y}^{q} \approx m_{u_{i j}} \bar{u}_{R_{i}} u_{L_{j}}+m_{d_{i j}} \bar{d}_{R_{i}} d_{L_{j}}+\text { h.c. } \\
& -\mathcal{L}_{Y}^{\ell} \approx m_{\ell_{i j}} \bar{e}_{R_{i}} \ell_{L_{j}}+\text { h.c. } \\
& -\mathcal{L}_{Y}^{m i x} \approx f_{i j} \overline{d_{R_{i}}} v_{L_{j}}\left(c_{a_{1}} A_{1}^{*}+s_{a_{1}} B_{1}^{*}\right) \\
& \quad-\frac{g_{i j}}{\sqrt{2}} \overline{d_{L_{i}}^{c}} v_{L_{j}}\left(-s_{a_{1}} A_{1}+c_{a_{1}} B_{1}\right) \\
& -f_{i j} \overline{d_{R_{i}}} \ell_{L_{j}}\left(c_{a_{2}} A_{2}+s_{a_{2}} B_{2}\right) \\
& \quad-\frac{g_{i j} \overline{u_{L_{i}}^{c}} \ell_{L_{j}}\left(-s_{a_{1}} A_{1}+c_{a_{1}} B_{1}\right)}{} \quad-g_{i j} \overline{d_{L_{i}}^{c}} \ell_{L_{j}} \delta_{4 / 3}+g_{i j} \overline{u_{L_{i}}^{c}} v_{L_{j}}\left(-s_{a_{2}} A_{2}^{*}+c_{a_{2}} B_{2}^{*}\right)+\text { h.c., }
\end{aligned}
$$

where we define $m_{u_{i j}} \equiv \frac{v y_{i j}^{u}}{\sqrt{2}}, m_{d_{i j}} \equiv \frac{v y_{i j}^{d}}{\sqrt{2}}$, and $m_{\ell_{i j}} \equiv \frac{v h_{i j}}{\sqrt{2}}$.

The next task is to determine the matrices of $y^{u}, y^{d}, h, f, g$ via modular $A_{4}$ symmetry. In the quark sector, we assign $Q_{L}$ to be 3 and $-2, \bar{u}_{R}$ to be $\left\{1,1^{\prime \prime}, 1^{\prime}\right\}$ and -4 , and $\bar{d}_{R}$ to be $\left\{1,1^{\prime \prime}, 1^{\prime}\right\}$ and 0 under $A_{4}$ and $-k$, respectively. This assignment is the same as the one in Ref. [45], and it is already 
Table 1 Charge assignments of the LQ bosons $\eta$ and $\Delta$ under $S U(3)_{C} \times$ $S U(2)_{L} \times U(1)_{Y} \times A_{4}$ where $k_{I}$ is the number of modular weight

\begin{tabular}{lll}
\hline & $\eta$ & $\Delta$ \\
\hline$S U(3)_{C}$ & $\mathbf{3}$ & $\overline{\mathbf{3}}$ \\
$S U(2)_{L}$ & $\mathbf{2}$ & $\mathbf{3}$ \\
$U(1)_{Y}$ & $\frac{1}{6}$ & $\frac{1}{3}$ \\
$A_{4}$ & $\mathbf{1}$ & $\mathbf{1}$ \\
$-k_{I}$ & -2 & -2 \\
\hline
\end{tabular}

known that allowed region [30]. Thus, we will work on the same $\tau$ region of the lepton sector in our numerical analysis. The up-type quark mass matrix is written as:

$$
\begin{aligned}
y^{u}= & \left(\begin{array}{ccc}
a_{u} & 0 & 0 \\
0 & a_{c} & 0 \\
0 & 0 & a_{t}
\end{array}\right)\left[\left(\begin{array}{lll}
f_{1} & f_{3} & f_{2} \\
f_{2} & f_{1} & f_{3} \\
f_{3} & f_{2} & f_{1}
\end{array}\right)\right. \\
& \left.+\left(\begin{array}{ccc}
g_{u 1} & 0 & 0 \\
0 & g_{u 2} & 0 \\
0 & 0 & g_{u 3}
\end{array}\right)\left(\begin{array}{lll}
f_{1}^{\prime} & f_{3}^{\prime} & f_{2}^{\prime} \\
f_{2}^{\prime} & f_{1}^{\prime} & f_{3}^{\prime} \\
f_{3}^{\prime} & f_{2}^{\prime} & f_{1}^{\prime}
\end{array}\right)\right],
\end{aligned}
$$

where $Y_{3}^{(6)} \equiv\left[f_{1}, f_{2}, f_{3}\right]^{T}$ and $Y_{3^{\prime}}^{(6)} \equiv\left[f_{1}^{\prime}, f_{2}^{\prime}, f_{3}^{\prime}\right]^{T}$, $g_{u 1}=\alpha_{u}^{\prime} / \alpha_{u}, g_{u 2}=\beta_{u}^{\prime} / \beta_{u}$ and $g_{u 3}=\gamma_{u}^{\prime} / \gamma_{u}$ are complex parameters, and $a_{u}, a_{c}$ and $a_{t}$ can be used to fit the masses of up quarks. The explicit forms of $f_{i}$ and $f_{i}^{\prime}$ are summarized in Appendix. Then $m_{u}$ is diagonalized by two unitary matrices as $D_{u}=V_{u_{R}}^{\dagger} m_{u} V_{u_{L}}$, where $D_{u} \equiv \operatorname{diag}\left(m_{u}, m_{c}, m_{t}\right)$ is mass eigenvalues. Therefore, we find $\left|D_{u}\right|^{2}=V_{u_{L}}^{\dagger} m_{u}^{\dagger} m_{u} V_{u_{L}}$.

On the other hand, the down-type quark mass matrix is given as:

$$
y^{d}=\left(\begin{array}{ccc}
a_{d} & 0 & 0 \\
0 & a_{s} & 0 \\
0 & 0 & a_{b}
\end{array}\right)\left(\begin{array}{lll}
y_{1} & y_{3} & y_{2} \\
y_{2} & y_{1} & y_{3} \\
y_{3} & y_{2} & y_{1}
\end{array}\right)
$$

where and $a_{d}, a_{s}$ and $a_{b}$ can be used to fit the masses of down quarks, and $Y_{3}^{(2)} \equiv\left[y_{1}, y_{2}, y_{3}\right]^{T}$ in Appendix. Then $m_{d}$ is diagonalized by two unitary matrices as $D_{d}=V_{d_{R}}^{\dagger} m_{d} V_{d_{L}}$, where $D_{d} \equiv \operatorname{diag}\left(m_{d}, m_{s}, m_{b}\right)$ is mass eigenvalues. Therefore, we find $\left|D_{d}\right|^{2}=V_{d_{L}}^{\dagger} m_{d}^{\dagger} m_{d} V_{d_{L}}$. Finally, we get the observable mixing matrix $V_{C K M}$ as follows:

$V_{C K M}=V_{u_{L}}^{\dagger} V_{d_{L}}$

\subsection{Lepton sector}

Now let us move on the lepton sector. We assign $L_{L}$ to be 3 and -2 and $\bar{e}_{R}$ to be $\left\{1,1^{\prime \prime}, 1^{\prime}\right\}$ and 0 under $A_{4}$ and $-k$, respectively. Here, both of the leptoquark scalars are assigned to be true $A_{4}$ singlets with -2 modular weight. The assignments of $A_{4}$ and $-k$ are also summarized in Tables 1 and
2. Under these assignments, we can write down the concrete matrices as follows:

$$
\begin{aligned}
h= & {\left[\begin{array}{ccc}
a_{\ell} & 0 & 0 \\
0 & b_{\ell} & 0 \\
0 & 0 & c_{\ell}
\end{array}\right]\left[\begin{array}{lll}
y_{1} & y_{3} & y_{2} \\
y_{2} & y_{1} & y_{3} \\
y_{3} & y_{2} & y_{1}
\end{array}\right], } \\
f= & {\left[\begin{array}{lll}
a_{\eta} & 0 & 0 \\
0 & b_{\eta} & 0 \\
0 & 0 & c_{\eta}
\end{array}\right]\left[\begin{array}{lll}
y_{1}^{\prime} & y_{3}^{\prime} & y_{2}^{\prime} \\
y_{2}^{\prime} & y_{1}^{\prime} & y_{3}^{\prime} \\
y_{3}^{\prime} & y_{2}^{\prime} & y_{1}^{\prime}
\end{array}\right], } \\
g= & a Y_{1}^{(6)}\left[\begin{array}{lll}
1 & 0 & 0 \\
0 & 0 & 1 \\
0 & 1 & 0
\end{array}\right]+\frac{b}{3}\left[\begin{array}{lll}
2 f_{1} & -f_{3}-f_{2} \\
-f_{3} & 2 f_{2} & -f_{1} \\
-f_{2}-f_{1} & 2 f_{3}
\end{array}\right] \\
& +\frac{c}{2}\left[\begin{array}{ccc}
0 & f_{3} & -f_{2} \\
-f_{3} & 0 & f_{1} \\
f_{2} & -f_{1} & 0
\end{array}\right] \\
& +\frac{b^{\prime}}{3}\left[\begin{array}{ccccc}
2 f_{1}^{\prime} & -f_{3}^{\prime} & -f_{2}^{\prime} \\
-f_{3}^{\prime} & 2 f_{2}^{\prime} & -f_{1}^{\prime} \\
-f_{2}^{\prime} & -f_{1}^{\prime} & 2 f_{3}^{\prime}
\end{array}\right]+\frac{c}{2}\left[\begin{array}{ccc}
0 & f_{3}^{\prime} & -f_{2}^{\prime} \\
f_{3}^{\prime} & 0 & f_{1}^{\prime} \\
f_{2}^{\prime} & -f_{1}^{\prime} & 0
\end{array}\right]
\end{aligned}
$$

where $Y_{3}^{(4)} \equiv\left[y_{1}^{\prime}, y_{2}^{\prime}, y_{3}^{\prime}\right]^{T}$ in Appendix.

The charged-lepton sector after spontaneous symmetry breaking is given by

$-\mathcal{L}_{Y}^{\ell}=v \frac{h_{i j}}{\sqrt{2}} \ell_{L_{i}} e_{R_{j}}+$ h.c..

Then $m_{\ell}$ (三vh/ $\sqrt{2}$ ) is diagonalized by two unitary matrices as $D_{\ell}=V_{\ell_{R}}^{\dagger} m_{\ell} V_{\ell_{L}}$, where $D_{\ell} \equiv \operatorname{diag}\left(m_{e}, m_{\mu}, m_{\tau}\right)$ is mass eigenvalues. Therefore, we find $\left|D_{\ell}\right|^{2}=V_{\ell_{L}}^{\dagger} m_{\ell}^{\dagger} m_{\ell} V_{\ell_{L}}$. The active neutrino mass matrix $m_{v}$ is given at one-loop level through the following interactions:

$$
\begin{aligned}
-L_{Y}^{\ell}= & F_{a j} \overline{d_{R_{a}}^{\prime}} v_{L_{j}}\left(c_{a_{1}} A_{1}+s_{a_{1}} B_{1}\right) \\
& -G_{a j} \overline{d_{L_{a}}^{\prime c}} v_{L_{j}}\left(-s_{a_{1}} A_{1}+c_{a_{1}} B_{1}\right),
\end{aligned}
$$

where $F \equiv V_{d_{R}}^{\dagger} f$ and $G \equiv V_{d_{L}}^{T} g$ and $d^{\prime}$ is mass eigenstate. Then, the neutrino masss matrix in Fig. 1 is given at one-loop level as follows:

$$
\begin{aligned}
\left(m_{\nu}\right)_{i j}= & s_{2 a_{1}} \frac{3}{4(4 \pi)^{2}}\left[1-\frac{m_{A_{1}}^{2}}{m_{B_{1}}^{2}}\right] \\
& \times \sum_{i=1}^{3}\left[F_{i a}^{T} D_{d_{a}} G_{a j}+G_{i a}^{T} D_{d_{a}} F_{a j}\right] F_{I}\left(r_{A_{1}}, r_{D_{d_{i}}}\right)
\end{aligned}
$$

$F_{I}\left(r_{1}, r_{2}\right)=\frac{r_{1}\left(r_{2}-1\right) \ln r_{1}-r_{2}\left(r_{1}-1\right) \ln r_{2}}{\left(r_{1}-1\right)\left(r_{2}-1\right)\left(r_{1}-r_{2}\right)}, \quad\left(r_{1} \neq 1\right)$, 
Table 2 Charge assignments of the SM fermions under $S U(3)_{C} \times S U(2)_{L} \times U(1)_{Y} \times A_{4}$ where $k_{I}$ is the number of modular weight

\begin{tabular}{|c|c|c|c|c|c|}
\hline & \multicolumn{5}{|c|}{ Fermions } \\
\hline & $\overline{Q_{L}}$ & $\bar{u}_{R}$ & $d_{R}$ & $L_{L}$ & $\bar{e}_{R}$ \\
\hline$S U(3)_{C}$ & 3 & $\overline{3}$ & $\overline{3}$ & 1 & 1 \\
\hline$S U(2)_{L}$ & 2 & 1 & 1 & 2 & 1 \\
\hline$U(1)_{Y}$ & $\frac{1}{6}$ & $-\frac{2}{3}$ & $\frac{1}{3}$ & $-\frac{1}{2}$ & 1 \\
\hline$A_{4}$ & 3 & $1,1^{\prime \prime}, 1^{\prime}$ & $1,1^{\prime \prime}, 1^{\prime}$ & 3 & $1,1^{\prime \prime}, 1^{\prime}$ \\
\hline$-k_{I}$ & -2 & -4 & 0 & -2 & 0 \\
\hline
\end{tabular}

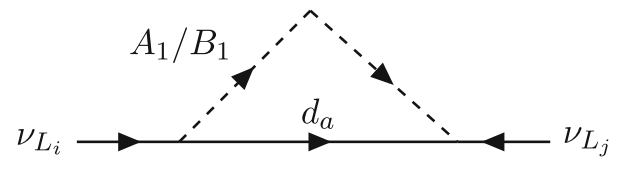

Fig. 1 One-loop diagrams for generating the neutrino mass matrix

where we define $r_{A_{1}} \equiv\left(m_{A_{1}} / m_{B_{1}}\right)^{2}$ and $r_{D_{d_{i}}} \equiv\left(D_{d_{i}} / m_{B_{1}}\right)^{2}$. $m_{v}$ is diagonalzied by a unitary matrix $V_{v} ; D_{v} \equiv V_{v}^{T} m_{\nu} V_{v}$. Here, we define a modified neutrino mass matrix as $\tilde{m}_{v} \equiv$ $m_{v} / s_{2 a_{1}}$. Then, we rewrite this diagonalization in terms of the modified form $\tilde{D}_{v} \equiv V_{v}^{T} \tilde{m}_{v} V_{v}$. Thus, we fix $s_{2 a_{1}}$ by

$(\mathrm{NH}): s_{2 a_{1}}^{2}=\frac{\left|\Delta m_{\mathrm{atm}}^{2}\right|}{\tilde{D}_{v_{3}}^{2}-\tilde{D}_{\nu_{1}}^{2}}, \quad(\mathrm{IH}): s_{2 a_{1}}^{2}=\frac{\left|\Delta m_{\mathrm{atm}}^{2}\right|}{\tilde{D}_{\nu_{2}}^{2}-\tilde{D}_{\nu_{3}}^{2}}$,

where $\tilde{m}_{v}$ is diagonalized by $V_{v}^{\dagger}\left(\tilde{m}_{v}^{\dagger} \tilde{m}_{v}\right) V_{v}=\left(\tilde{D}_{v_{1}}^{2}, \tilde{D}_{v_{2}}^{2}\right.$, $\left.\tilde{D}_{v_{3}}^{2}\right)$ and $\Delta m_{\mathrm{atm}}^{2}$ is the atmospheric neutrino mass-squared difference. Here, $\mathrm{NH}$ and $\mathrm{IH}$ respectively stand for the normal hierarchy and the inverted hierarchy. Subsequently, the solar neutrino mass-squared difference is depicted in terms of $s_{2 a_{1}}$ as follows:

$\Delta m_{\mathrm{sol}}^{2}=s_{2 a_{1}}^{2}\left(\tilde{D}_{\nu_{2}}^{2}-\tilde{D}_{\nu_{1}}^{2}\right)$.

This should be within the experimental value, where we adopt NuFit 5.0 [109] to our numerical analysis later. The neutrinoless double beta decay is also given by

$$
\begin{aligned}
\left\langle m_{e e}\right\rangle= & s_{2 a_{1}} \mid \tilde{D}_{\nu_{1}} \cos ^{2} \theta_{12} \cos ^{2} \theta_{13} \\
& +\tilde{D}_{\nu_{2}} \sin ^{2} \theta_{12} \cos ^{2} \theta_{13} e^{i \alpha_{2}} \\
& +\tilde{D}_{\nu_{3}} \sin ^{2} \theta_{13} e^{i\left(\alpha_{3}-2 \delta_{C P}\right)} \mid,
\end{aligned}
$$

which may be able to observed by KamLAND-Zen in future [110]. The observed mixing matrix of lepton sector [111] is given by $V_{\mathrm{PMNS}} \equiv V_{\ell_{L}}^{\dagger} V_{\nu}$.

\section{Numerical analysis}

Here, we perform numerical analysis. Before searching for allowed region, we fix some mass parameters as $m_{A_{2}}=m_{A_{1}}$ and $m_{B_{2}}=m_{\delta}=m_{B_{1}}$, where we require degenerate masses for the components of $\eta$ and $\Delta$ to suppress the oblique parameters $\Delta S$ and $\Delta T$. Notice here that our theoretical parameters $a_{u, c, t}, a_{d, s, b}, a_{\ell}, b_{\ell}, c_{\ell}$ are used to determined the experimental masses for quarks and charged-leptons. Thus, only the following input parameters are randomly selected in the range of

$$
\begin{aligned}
& \left(m_{A_{1}}, m_{B_{1}}\right) \in[1,100] \mathrm{TeV}, \\
& \left|g_{u 1, u 2, u 3}\right| \in\left[10^{-5}, 1.5\right], \\
& \quad\left(\left|a_{\eta}\right|,\left|b_{\eta}\right|,\left|c_{\eta}\right|,|a|,|b|,|c|,\left|b^{\prime}\right|,\left|c^{\prime}\right|\right) \in\left[10^{-5}, 10\right] .
\end{aligned}
$$

Above the range, we have numerical analysis in cases for quark and lepton, where experimental data in the quark sector should be within the range at $3 \sigma$. While the one in the lepton sector is discussed in the range within $3 \sigma$ (yellow dots) and $5 \sigma$ (red dots) applying $\chi^{2}$ analysis in Nufit 5.0.

\section{$3.1 \mathrm{NH}$}

For NH case, we show our results of lepton sector in Figs. 2, 3, 4, 5. In Fig. 2, allowed value of $\tau$ is shown where yellow(red) points present the values within 3(5) $\sigma$. One finds that allowed space is rather localized. Especially, the region at nearby $\tau \sim 1.75 i$ would be interesting since it is close to the fixed point that has a remnant $Z_{3}$ symmetry. In Fig. 3, we demonstrate allowed region of $\delta_{C P}$ in terms of $\sum m_{i}$. $\sum m_{i}$ is rather localized at $0.06-0.08 \mathrm{eV}$ while whole the region is allowed for $\delta_{C P}$. Moreover, almost all the points are within the cosmological constraint $\sim 0.12 \mathrm{eV}$ [112]. In Fig. 4, we present allowed region of neutrinoless double beta decay $\left\langle m_{e e}\right\rangle$ in terms of the lightest neutrino mass $m_{1} .\left\langle m_{e e}\right\rangle$ is allowed up to $0.025 \mathrm{eV}$ while $m_{1}$ is allowed up to $0.0025 \mathrm{eV}$. Moreover, allowed region of $m_{1}$ is localized at around $10^{-6} \mathrm{eV}$ indicating tiny mass of the lightest neutrino mass. In Fig. 5, we 


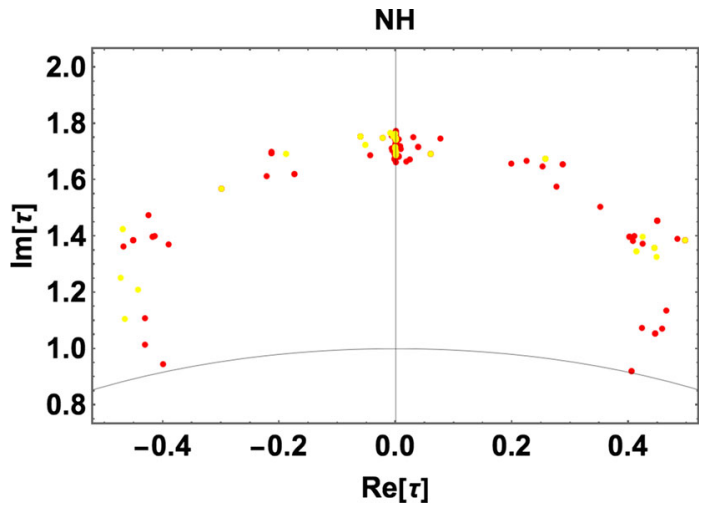

Fig. 2 Allowed value of $\tau$. Yellow and red points present the values within 3 and $5 \sigma$

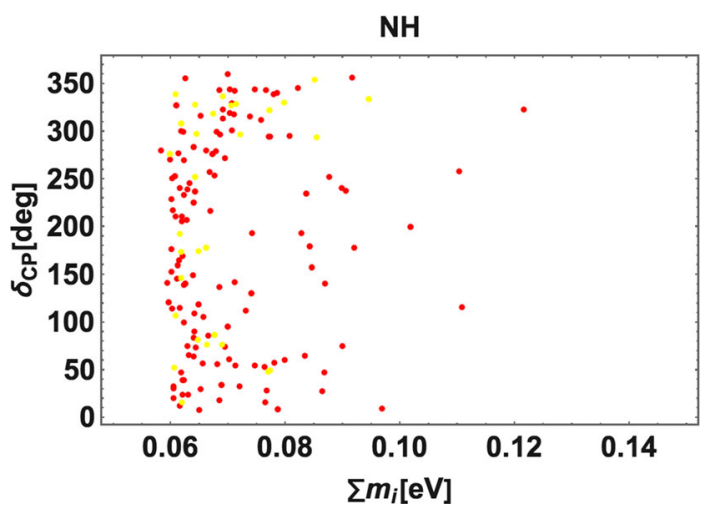

Fig. 3 Allowed region of $\delta_{C P}$ in terms of $\sum m_{i}$

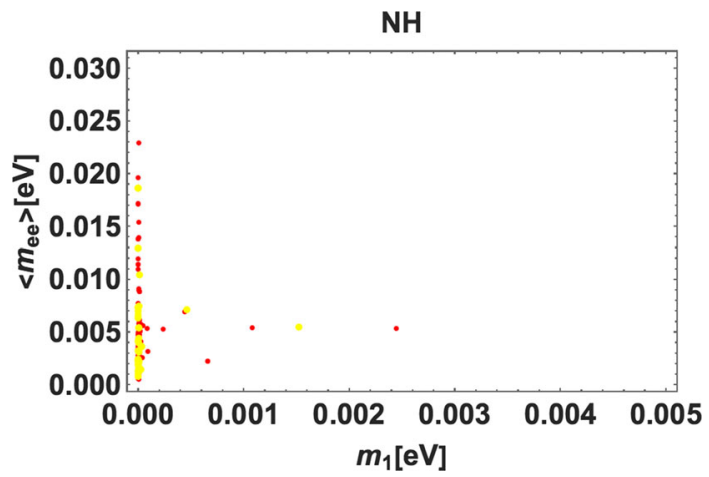

Fig. 4 Allowed region of the mass of neutrinoless double beta decay in terms of the lightest neutrino mass

depict allowed region of Majorana phases $\alpha_{21}$ and $\alpha_{31}$. Both are allowed for whole the region but there would be tendency that $\alpha_{21}$ is localized at around $180^{\circ}$.

In addition to the lepton sector, we will search for our allowed region of quark sector in Figs. 6, 7, 8. Here, the dotted red line at $3 \sigma$ interval while the black line is best fit value. And the yellow(red) points correspond to 3(5) $\sigma$ of the lepton sector where $\tau$ is commonly used.

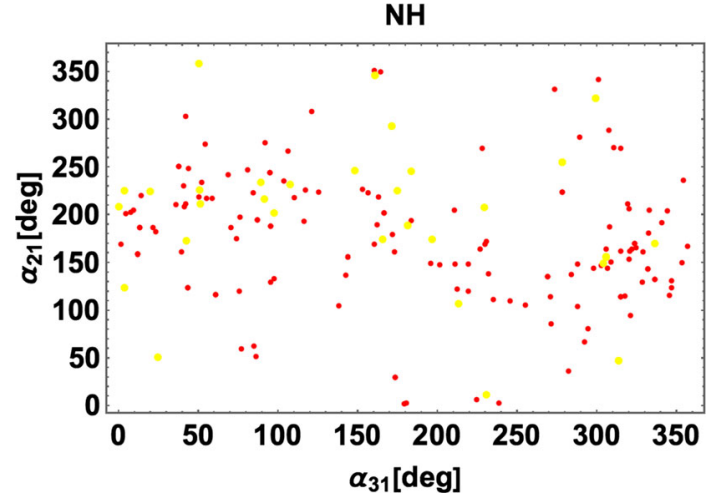

Fig. 5 Allowed region of Majorana phases $\alpha_{21}$ in terms of $\alpha_{31}$

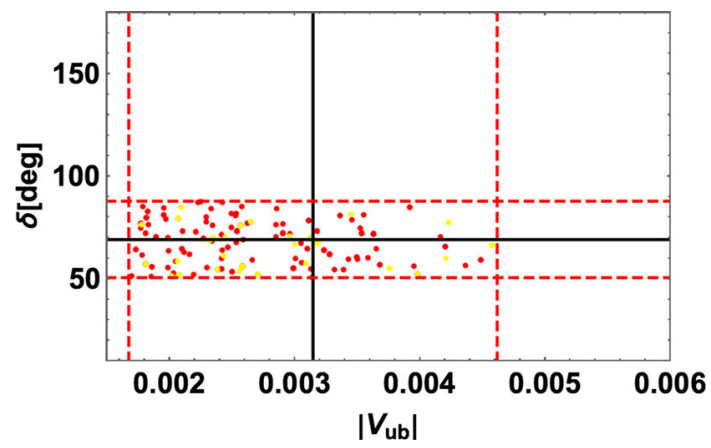

Fig. 6 The CP phase of quark $\delta$ versus $(1,3)$ component of CKM matrix. The red dashed lines represent $3 \sigma$ experimental bounds

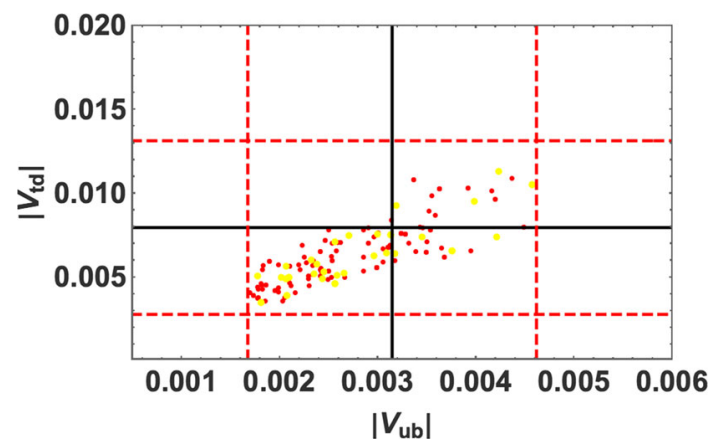

Fig. $7\left|V_{u b}\right|$ versus $\left|V_{t d}\right|$

In Fig. 6, we show the CP phase of quark $\delta$ in term of $(1,3)$ component of CKM matrix; $\left|V_{u b}\right|$, and find whole the region is allowed at $3 \sigma$ interval. In Fig. 7, we show $\left|V_{u b}\right|$ and $\left|V_{t d}\right|$, and found that there is a weak linearly correlation between them. In Fig. 8, we show $\left|V_{c b}\right|$ and $\left|V_{u b}\right|$, and find that there is also a weak linear correlation between them.

Bench mark point for $\mathrm{NH}$ : We also give a benchmark point to satisfy the quark and lepton masses and mixings as well as phases in the left sides of Tables 3 and 4 , where we extracted a value at nearby $\tau=1.75 i$. The corresponding lepton and neutrino mixings are given by 


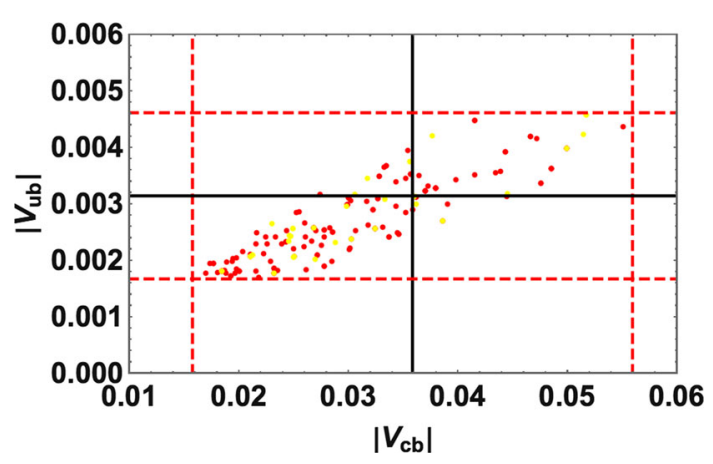

Fig. $8\left|V_{c b}\right|$ versus $\left|V_{u b}\right|$

$$
\begin{aligned}
V_{\ell_{L}}= & {\left[\begin{array}{ccc}
-0.75+0.00017 i & 0.35-0.000026 i & -0.56 \\
0.20-0.000012 i & -0.68+0.000074 i & -0.70-0.000040 i \\
-0.63+0.00013 i & -0.64+0.000077 i & 0.44+0.000044 i
\end{array}\right], } \\
V_{v} & =\left[\begin{array}{ccc}
-0.91-0.20 i & 0.26-0.075 i & -0.22-0.027 i \\
0.23+0.018 i & -0.045-0.28 i & -0.87-0.33 i \\
-0.13+0.24 i & -0.25+0.88 i & -0.25-0.15 i
\end{array}\right]
\end{aligned}
$$

And the quark mixings are given by

$$
\begin{aligned}
V_{u_{L}}= & {\left[\begin{array}{ccc}
-0.75-0.057 i & 0.41+0.24 i & -0.46+0.0034 i \\
-0.62+0.017 i & -0.31-0.068 i & 0.71-0.0045 i \\
0.19-0.071 i & 0.76+0.30 i & 0.53-0.0015 i
\end{array}\right], } \\
V_{d_{L}}= & {\left[\begin{array}{ccc}
-0.64+0.000077 i & -0.63+0.00013 i & -0.44-0.000044 i \\
-0.68+0.000074 i & 0.20-0.000012 i & 0.70+0.000040 i \\
0.35-0.000026 i & -0.75+0.00017 i & 0.56
\end{array}\right] . }
\end{aligned}
$$

\section{$3.2 \mathrm{IH}$}

In case of IH, we obtain less allowed parameter points compared to the case of $N H$ since it is more difficult to fit the data. Since there are no points within $3 \sigma$ region but few points within $5 \sigma$ region, we will explain the tendency instead of showing scattering plots. The value of $\tau$ is interestingly localized at nearby two fixed points $i$ and $1.74 i$, each of which has remnant symmetry of $Z_{2}$ and $Z_{3}$. $\sum m_{i}$ is localized at 0.10 $0.12 \mathrm{eV}$ while $\delta_{C P}$ is allowed for the range of $150^{\circ}-360^{\circ}$. Moreover, almost all the points are within the cosmological constraint $\sim 0.12 \mathrm{eV}$ that is similar to the NH case. $\left\langle m_{e e}\right\rangle$ is localized at around $0.016-0.024 \mathrm{eV}$ while $m_{1}$ is allowed up to $1.2 \times 10^{-4} \mathrm{eV}$. Moreover, $m_{1}$ is also localized at around $10^{-6}$ $\mathrm{eV} . \alpha_{21}$ is localized at around $180^{\circ}$, while $\alpha_{21}$ is allowed for the range of $100^{\circ}-360^{\circ}$.

In addition to the lepton sector, we discuss our allowed region of quark sector. Even though the allowed points are not so many, we might say something from our analysis as follows. As for the CP phase of quark $\delta$ in term of $(1,3)$ component of CKM matrix; $\left|V_{u b}\right|$, we found whole the region is allowed at $3 \sigma$ interval. As for $\left|V_{u b}\right|$ and $\left|V_{t d}\right|$, we find that there is a weak linearly correlation between them. As for $\left|V_{c b}\right|$ and $\left|V_{u b}\right|$, we find that there is also a weak linear correlation between them.

Bench mark point for IH: We give two interesting benchmark points; $\tau \approx 1.06 i, 1.76 i$ to satisfy the quark and lepton masses and mixings as well as phases in the center and right sides of Tables 3 and 4 . The lepton and neutrino mixings are given by

$$
\begin{aligned}
\tau & \approx 1.06 i: \\
V_{\ell_{L}} & =\left[\begin{array}{ccc}
-0.65+0.0068 i & 0.72+0.00024 i & -0.25+0.00061 i \\
-0.47+0.0067 i & -0.64+0.0012 i & -0.61+0.00072 i \\
-0.60+0.0068 i & -0.28+0.00098 i & 0.75+0.000042 i
\end{array}\right] \\
V_{\nu} & =\left[\begin{array}{ccc}
-0.63+0.12 i & 0.053-0.029 i & -0.13-0.75 i \\
0.090+0.11 i & 0.14+0.98 i & 0.015-0.089 i \\
-0.75+0.10 i & 0.12+0.097 i & 0.068+0.63 i
\end{array}\right] \\
\tau & \approx 1.76 i: \\
V_{\ell_{L}} & =\left[\begin{array}{ccc}
-0.21-0.000031 i & 0.80-0.0015 i & 0.56+0.000052 i \\
0.63-0.00065 i & -0.33+0.00026 i & 0.70+0.00032 i \\
0.75-0.00091 i & 0.50-0.0010 i & -0.44-0.00036 i
\end{array}\right], \\
V_{\nu} & =\left[\begin{array}{ccc}
-0.010+0.016 i & 0.063-0.098 i & -0.87+0.48 i \\
0.28-0.037 i & -0.77+0.56 i & -0.12+0.014 i \\
0.53+0.80 i & 0.25+0.13 i & 0.016+0.0094 i
\end{array}\right]
\end{aligned}
$$

The quark mixings are given by

$$
\begin{aligned}
\tau & \approx 1.06 i: \\
V_{u_{L}}= & {\left[\begin{array}{ccc}
-0.59+0.26 i & -0.18-0.067 i & -0.71-0.23 i \\
-0.55+0.21 i & -0.50-0.030 i & 0.61+0.18 i \\
-0.41+0.28 i & 0.84-0.062 i & 0.20+0.080 i
\end{array}\right], } \\
V_{d_{L}}= & {\left[\begin{array}{ccc}
-0.60+0.0068 i & -0.28+0.00096 i & -0.75-0.000044 i \\
-0.47+0.0067 i & -0.64+0.0011 i & 0.61-0.00072 i \\
-0.65+0.0068 i & 0.72+0.00022 i & 0.25-0.00061 i
\end{array}\right], } \\
\tau & \approx 1.76 i: \\
V_{u_{L}}= & {\left[\begin{array}{ccc}
-0.76-0.042 i & 0.43+0.199 & -0.45+0.0188 i \\
-0.62+0.015 i & -0.33-0.052 i & 0.71-0.026 i \\
0.19-0.054 i & 0.78+0.23 i & 0.54-0.014 i
\end{array}\right], } \\
V_{d_{L}}= & {\left[\begin{array}{ccc}
-0.64+0.00066 i & -0.63+0.0011 i & -0.44-0.00039 i \\
-0.68+0.00064 i & 0.21-0.00013 i & 0.70+0.00037 i \\
0.35-0.00023 i & -0.75+0.0015 i & 0.56+0.000089 i
\end{array}\right] . }
\end{aligned}
$$

\section{Conclusions}

We have proposed a LQ model to explain the masses and mixings for quark and lepton, introducing modular $A_{4}$ symmetry. Due to nature of LQ model that lepton(quark) directly connects to the quark(lepton) via LQ, a single modulus number has to be applied that leads to a good motivation towards unification of quark and lepton flavor in $A_{4}$ modular symme- 
Table 3 Numerical values of parameters and observables at the best fit points of $\mathrm{NH}$ and $\mathrm{IH}$

\begin{tabular}{|c|c|c|c|}
\hline Lepton & $\mathrm{NH}(\tau \approx 1.75 i)$ & $\mathrm{IH}(\tau \approx 1.06 i)$ & $\mathrm{IH}(\tau \approx 1.76 i)$ \\
\hline$\tau$ & $-0.0000945+1.75 i$ & $-0.000689+1.06 i$ & $-0.000829+1.76 i$ \\
\hline$a_{\eta}$ & $-0.23-1.4 i$ & $-0.31+0.013 i$ & $-4.1+4.3 i$ \\
\hline$b_{\eta}$ & $-0.38+1.3 i$ & $-0.045-0.027 i$ & $-0.0014+0.0032 i$ \\
\hline$c_{\eta}$ & $0.0077-0.031 i$ & $0.0014-0.000047 i$ & $-0.0023+0.0035 i$ \\
\hline$a$ & $0.00016+0.00011 i$ & $3.0+0.98 i$ & $0.0085+0.025 i$ \\
\hline$b$ & $-0.017+0.0013 i$ & $0.00014+0.00015 i$ & $-0.096+0.041 i$ \\
\hline$c$ & $-0.00030-0.00010 i$ & $0.0056-0.0021 i$ & $-1.8+4.5 i$ \\
\hline$b^{\prime}$ & $0.00014-0.000016 i$ & $0.000024+0.000016 i$ & $-0.00011+0.00011 i$ \\
\hline$c^{\prime}$ & $-0.21-0.27 i$ & $-0.15+0.031 i$ & $-0.0000034-0.000060 i$ \\
\hline$\left[\alpha_{e}, \beta_{e}, \gamma_{e}\right]$ & {$\left[0.0002,9.3 \times 10^{-7}, 0.003\right]$} & {$\left[0.0005,10^{-5}, 0.007\right]$} & {$\left[9.1 \times 10^{-7}, 1.9 \times 10^{-4}, 0.003\right]$} \\
\hline $\sin ^{2} \theta_{12}$ & 0.32 & 0.28 & 0.33 \\
\hline $\sin ^{2} \theta_{23}$ & 0.56 & 0.46 & 0.58 \\
\hline $\sin ^{2} \theta_{13}$ & 0.024 & 0.024 & 0.022 \\
\hline$\delta_{C P}^{\ell}$ & $328^{\circ}$ & $170^{\circ}$ & $335^{\circ}$ \\
\hline$\left[\alpha_{21}, \alpha_{31}\right]$ & {$\left[169^{\circ}, 336^{\circ}\right]$} & {$\left[167^{\circ}, 159^{\circ}\right]$} & {$\left[157^{\circ}, 130^{\circ}\right]$} \\
\hline$\sum m_{i}$ & $0.071 \mathrm{eV}$ & $0.11 \mathrm{eV}$ & $0.11 \mathrm{eV}$ \\
\hline$s_{2 a_{1}}$ & $4.6 \times 10^{-9}$ & $5.7 \times 10^{-5}$ & $1.9 \times 10^{-9}$ \\
\hline$\left\langle m_{e e}\right\rangle$ & $3.2 \mathrm{meV}$ & $21 \mathrm{meV}$ & $19 \mathrm{meV}$ \\
\hline$\left[m_{A_{1}}, m_{B_{1}}\right]$ & {$[18,6.0] \mathrm{TeV}$} & {$[37,37] \mathrm{TeV}$} & {$[33,39] \mathrm{TeV}$} \\
\hline$\sqrt{\chi^{2}}$ & 2.9 & 4.8 & 4.5 \\
\hline
\end{tabular}

Table 4 Numerical values of parameters and observables at the best fit points of $\mathrm{NH}$ and $\mathrm{IH}$

\begin{tabular}{llll}
\hline Quark & $\mathrm{NH}(\tau \approx 1.75 i)$ & $\mathrm{IH}(\tau \approx 1.06 i)$ & $\mathrm{IH}(\tau \approx 1.76 i)$ \\
\hline$\tau$ & $-0.0000945+1.75 i$ & $-0.000689+1.06 i$ & $-0.000829+1.76 i$ \\
$a_{u}$ & $1.3 \times 10^{-7}$ & $8.2 \times 10^{-8}$ & $1.1 \times 10^{-5}$ \\
$a_{c}$ & $4.6 \times 10^{-5}$ & $4.2 \times 10^{-5}$ & 0.0007 \\
$a_{t}$ & 0.017 & 0.017 & 0.22 \\
$g_{u 1}$ & $0.00066+0.0016 i$ & $-0.00013+0.013 i$ & $0.57-0.35 i$ \\
$g_{u 2}$ & $0.053+0.30 i$ & $0.094+0.24 i$ & $-0.060-0.41 i$ \\
$g_{u 3}$ & $0.11+0.0061 i$ & $0.091+0.018 i$ & $0.046+0.021 i$ \\
$a_{d}$ & 0.00018 & 0.00014 & 0.00047 \\
$a_{S}$ & $1.2 \times 10^{-5}$ & $8.7 \times 10^{-6}$ & $5.2 \times 10^{-5}$ \\
$a_{b}$ & 0.011 & 0.011 & 0.025 \\
$\left|V_{u s}\right|$ & 0.23 & 0.22 & 0.22 \\
$\left|V_{c b}\right|$ & 0.033 & 0.027 & 0.042 \\
$\left|V_{u b}\right|$ & 0.0031 & 0.0020 & 0.0039 \\
$\delta_{C P}$ & $58^{\circ}$ & $51^{\circ}$ & $83^{\circ}$ \\
\hline
\end{tabular}

try. After giving an assignment for quark sector to reproduce the experimental results at $3 \sigma$ interval, we have also constructed the lepton sector, where the neutrino mass matrix is induced at one-loop level running down quark sector, unified value of $\tau$ is used for quark and lepton.

Then, we have performed numerical analysis to search for allowed region satisfying experimental measurements for both quark and lepton sector, depending on $\mathrm{NH}$ and $\mathrm{IH}$.
In case of $\mathrm{NH}$, we have found rather wide allowed space within $3 \sigma$ interval and obtained tendency of observables for quark and lepton. Especially, we have found allowed region at nearby $\tau=1.75 i$ that is close to the fixed point of $\tau=i \infty$. Thus, we have also shown a promising bench mark point at around the solution.

In case of $\mathrm{IH}$, we would not found the allowed region within $3 \sigma$ interval, but found within $5 \sigma$ interval. Although 
the number of allowed point is few, we have found all the allowed regions are localized at nearby $\tau=i, 1.76 i$, both of which are nearby fixed points. We have shown them as benchmark points. These would be tested near future.

Before closing it is worthwhile mentioning on flavor physics and collider phenomenology of our model. On hadron collider experiments such as the LHC, lepto-quarks can be pair produced via strong interactions and lower limits of their masses are given as $\mathcal{O}(1) \mathrm{TeV}$ depending on its decay modes [113-115]. In addition the most striking signature would arise from the lepton flavor violating process at the LHC via lepto-quarks in the t-channel, $q \bar{q} \rightarrow \ell^{\prime+} \ell^{-}$, with final states such as $e^{ \pm} \mu^{\mp}, e^{ \pm} \tau^{\mp}, \mu^{ \pm} \tau^{\mp}$. Taking $g=$ $f \approx 0.1$ and $1 \mathrm{TeV}$ lepto-quark mass, we find 6 event rate for $d \bar{d} \rightarrow e^{ \pm} \tau^{\mp}$, which is maximum, at the $13 \mathrm{TeV} \mathrm{LHC}$ with $300 \mathrm{fb}^{-1}$ luminosity; more discussion can be found in ref. [14]. For flavor physics, whenever considering radiative seesaw models, we have to consider lepton flavor violations, especially, $\mu \rightarrow e \gamma$. This gives the most stringent constraint on Yukawa couplings and masses of mediated particles which are lepto-quarks in our case. For our model, Yukawa couplings $f, g$ can be order 1 , since the lepto-quark masses has to be larger than $1 \mathrm{TeV}$ from the collider analysis at LHC. Thus, our parameters are totally safe from this constraint including other $\tau \rightarrow e(\mu) \gamma$ modes. Moreover from interactions associated with the Yukawa coupling $g$, we may find an interesting effects on $b \rightarrow s \mu \bar{\mu}$ anomaly, which can be characterized by Wilson coefficients $C_{9}=-C_{10}$ of six dimensional effective Hamiltonian $\left[\left(\bar{s} \gamma_{\mu} P_{L} b\right)\left(\bar{\mu} \gamma^{\mu} \mu\right)-\left(\bar{s} \gamma_{\mu} P_{L} b\right)\left(\bar{\gamma}^{\mu} \gamma_{5} \mu\right)\right]$. Experimental results tell us $\Delta C_{9} \approx-1$ as new physics contribution. To get this order, we need $g \sim 0.1$, supposing 1 $\mathrm{TeV}$ of lepto-quark mass. However since $\Delta C_{10}$ also contributes to the process of $b \rightarrow \mu \bar{\mu}$ that gives a constraint of $\Delta C_{10} \approx 0.1$. Thus, we would need to modify the model if one wants to get the sizable anomaly of $b \rightarrow s \mu \bar{\mu}$. Yukawa coupling $g$ (as well as $f$ ) also receives constraints from neutral meson mixings such as $\mathrm{K}$-short and $\mathrm{K}$-long at one-loop box diagram. Typically, $g(f)$ should be less than $\sim 0.1$ at 1 $\mathrm{TeV}$ of lepto-quark mass. We have a source of muon $g-2$ from both the Yukawa couplings $g$ and $f$ at one-loop level. However, assuming $g=f \approx 0.1$ and $1 \mathrm{TeV}$ lepto-quark mass, the value of muon $g-2$ is at most $10^{-12}$ in our model that is far from the current experimental value $10^{-9}$. Thus, we need to improve this model such that it does not include chiral suppression. In conclusion, we need extension of the model to resolve flavor anomalies such as muon $g-2$ and $b \rightarrow s \mu \bar{\mu}$.

Acknowledgements This research was supported by an appointment to the JRG Program at the APCTP through the Science and Technology Promotion Fund and Lottery Fund of the Korean Government. This was also supported by the Korean Local Governments-Gyeongsangbuk-do Province and Pohang City (H.O.), European Regional Development Fund-Project Engineering Applications of Microworld Physics (Grant
No. CZ.02.1.01/0.0/0.0/16_019/0000766) (Y.O.). H. O. is sincerely grateful for the KIAS member.

Data Availability Statement This manuscript has no associated data or the data will not be deposited. [Authors' comment: Since our work is theoretical analysis we do not have specific data to provide.]

Open Access This article is licensed under a Creative Commons Attribution 4.0 International License, which permits use, sharing, adaptation, distribution and reproduction in any medium or format, as long as you give appropriate credit to the original author(s) and the source, provide a link to the Creative Commons licence, and indicate if changes were made. The images or other third party material in this article are included in the article's Creative Commons licence, unless indicated otherwise in a credit line to the material. If material is not included in the article's Creative Commons licence and your intended use is not permitted by statutory regulation or exceeds the permitted use, you will need to obtain permission directly from the copyright holder. To view a copy of this licence, visit http://creativecomm ons.org/licenses/by/4.0/.

Funded by $\mathrm{SCOAP}^{3}$.

\section{Appendix}

The modular forms of weight $2, Y_{\mathbf{3}}^{(2)}=\left[y_{1}, y_{2}, y_{3}\right]^{T}$, transforming as a triplet of $A_{4}$ is written in terms of Dedekind eta-function $\eta(\tau)$ and its derivative:

$$
\begin{aligned}
y_{1}(\tau)= & \frac{i}{2 \pi}\left(\frac{\eta^{\prime}(\tau / 3)}{\eta(\tau / 3)}+\frac{\eta^{\prime}((\tau+1) / 3)}{\eta((\tau+1) / 3)}\right. \\
& \left.+\frac{\eta^{\prime}((\tau+2) / 3)}{\eta((\tau+2) / 3)}-\frac{27 \eta^{\prime}(3 \tau)}{\eta(3 \tau)}\right), \\
y_{2}(\tau)= & \frac{-i}{\pi}\left(\frac{\eta^{\prime}(\tau / 3)}{\eta(\tau / 3)}+\omega^{2} \frac{\eta^{\prime}((\tau+1) / 3)}{\eta((\tau+1) / 3)}+\omega \frac{\eta^{\prime}((\tau+2) / 3)}{\eta((\tau+2) / 3)}\right), \\
y_{3}(\tau)= & \frac{-i}{\pi}\left(\frac{\eta^{\prime}(\tau / 3)}{\eta(\tau / 3)}+\omega \frac{\eta^{\prime}((\tau+1) / 3)}{\eta((\tau+1) / 3)}+\omega^{2} \frac{\eta^{\prime}((\tau+2) / 3)}{\eta((\tau+2) / 3)}\right), \\
\eta(\tau)= & q^{1 / 24} \Pi_{n=1}^{\infty}\left(1-q^{n}\right), \quad q=e^{2 \pi i \tau}, \quad \omega=e^{2 \pi i / 3}
\end{aligned}
$$

Then, any multiplets of higher weight are constructed by multiplication rules of $A_{4}$, and one finds the following :

$$
\begin{aligned}
Y_{\mathbf{1}}^{(4)} & =y_{1}^{2}+2 y_{2} y_{3}, \quad Y_{\mathbf{3}}^{(4)} \equiv\left[\begin{array}{l}
y_{1}^{\prime} \\
y_{2}^{\prime} \\
y_{3}^{\prime}
\end{array}\right]=\left[\begin{array}{l}
y_{1}^{2}-y_{2} y_{3} \\
y_{3}^{2}-y_{1} y_{2} \\
y_{2}^{2}-y_{1} y_{3}
\end{array}\right] \\
Y_{\mathbf{1}}^{(6)} & =y_{1}^{2}+y_{2}^{2}+y_{3}^{2}-3 y_{1} y_{2} y_{3} \\
Y_{\mathbf{3}}^{(6)} & \equiv\left[\begin{array}{l}
f_{1} \\
f_{2} \\
f_{3}
\end{array}\right]=\left[\begin{array}{l}
y_{1}^{3}+2 y_{1} y_{2} y_{3} \\
y_{1}^{2} y_{2}+2 y_{2}^{2} y_{3} \\
y_{1}^{2} y_{3}+2 y_{3}^{2} y_{2}
\end{array}\right] \\
Y_{\mathbf{3}^{\prime}}^{(6)} & \equiv\left[\begin{array}{l}
f_{1}^{\prime} \\
f_{2}^{\prime} \\
f_{3}^{\prime}
\end{array}\right]=\left[\begin{array}{l}
y_{3}^{3}+2 y_{1} y_{2} y_{3} \\
y_{3}^{2} y_{1}+2 y_{1}^{2} y_{2} \\
y_{3}^{2} y_{2}+2 y_{2}^{2} y_{1}
\end{array}\right]
\end{aligned}
$$




\section{References}

1. M. Bauer, M. Neubert, Phys. Rev. Lett. 116(14), 141802 (2016). $\quad$ https://doi.org/10.1103/PhysRevLett.116.141802. arXiv:1511.01900 [hep-ph]

2. C.H. Chen, T. Nomura, H. Okada, Phys. Rev. D 94(11), 115005 (2016). https://doi.org/10.1103/PhysRevD.94.115005. arXiv:1607.04857 [hep-ph]

3. E. Coluccio Leskow, G. D'Ambrosio, A. Crivellin, D. Müller, Phys. Rev. D 95(5), 055018 (2017). https://doi.org/10.1103/ PhysRevD.95.055018. arXiv:1612.06858 [hep-ph]

4. C.H. Chen, T. Nomura, H. Okada, Phys. Lett. B 774, 456-464 (2017). https://doi.org/10.1016/j.physletb.2017.10.005. arXiv:1703.03251 [hep-ph]

5. T. Nomura, H. Okada, arXiv:2104.03248 [hep-ph]

6. D. Zhang, arXiv:2105.08670 [hep-ph]

7. S. Sahoo, R. Mohanta, Phys. Rev. D 91(9), 094019 (2015). https:// doi.org/10.1103/PhysRevD.91.094019. arXiv:1501.05193 [hep$\mathrm{ph}]$

8. D. Becirevic, S. Fajfer, N. Kosnik, O. Sumensari, Phys. Rev. D 94(11), 115021 (2016). https://doi.org/10.1103/PhysRevD.94. 115021. arXiv:1608.08501 [hep-ph]

9. Y. Cai, J. Gargalionis, M.A. Schmidt, R.R. Volkas, JHEP 10, 047 (2017). https://doi.org/10.1007/JHEP10(2017)047. arXiv:1704.05849 [hep-ph]

10. Y. Sakaki, M. Tanaka, A. Tayduganov, R. Watanabe, Phys. Rev. D 88(9), 094012 (2013). https://doi.org/10.1103/PhysRevD.88. 094012. arXiv:1309.0301 [hep-ph]

11. D. Aristizabal Sierra, M. Hirsch, S.G. Kovalenko, Phys. Rev. D 77, 055011 (2008). https://doi.org/10.1103/PhysRevD.77. 055011. arXiv:0710.5699 [hep-ph]

12. I. de Medeiros Varzielas, G. Hiller, JHEP 06, 072 (2015). https:// doi.org/10.1007/JHEP06(2015)072. arXiv:1503.01084 [hep-ph]

13. G. Hiller, D. Loose, K. Schönwald, JHEP 12, 027 (2016). https:// doi.org/10.1007/JHEP12(2016)027. arXiv:1609.08895 [hep-ph]

14. K. Cheung, T. Nomura, H. Okada, Phys. Rev. D 94(11), 115024 (2016). https://doi.org/10.1103/PhysRevD.94.115024. arXiv:1610.02322 [hep-ph]

15. M. Huschle et al. [Belle Collaboration], Phys. Rev. D 92(7), 072014 (2015) https://doi.org/10.1103/PhysRevD.92.072014. arXiv: 1507.03233 [hep-ex]

16. J.P. Lees et al. [BaBar Collaboration], Phys. Rev. Lett. 109, 101802 (2012). https://doi.org/10.1103/PhysRevLett.109. 101802. arXiv:1205.5442 [hep-ex]

17. J.P. Lees et al. [BaBar Collaboration], Phys. Rev. D 88(7), 072012 (2013). https://doi.org/10.1103/PhysRevD.88.072012. arXiv:1303.0571 [hep-ex]

18. A. Abdesselam et al. [Belle Collaboration], arXiv:1603.06711 [hep-ex]

19. S. Hirose et al. [Belle], Phys. Rev. Lett. 118(21), 211801 (2017). https://doi.org/10.1103/PhysRevLett.118.211801. arXiv:1612.00529 [hep-ex]

20. R. Aaij et al. [LHCb Collaboration], Phys. Rev. Lett. 115, no. 11, 111803 (2015) Addendum: [Phys. Rev. Lett. 115, no. 15, 159901 (2015)] 10.1103/PhysRevLett.115.159901, 10.1103/PhysRevLett.115.111803 [arXiv:1506.08614 [hep-ex]]

21. R. Aaij et al. [LHCb], Phys. Rev. D 97(7), 072013 (2018). https:// doi.org/10.1103/PhysRevD.97.072013. arXiv:1711.02505 [hepex]

22. B. Abi et al. [Muon g-2], Phys. Rev. Lett. 126(14), 141801 (2021). https://doi.org/10.1103/PhysRevLett.126.141801. arXiv:2104.03281 [hep-ex]

23. R. Aaij et al. [LHCb], arXiv:2103.11769 [hep-ex]

24. F. Feruglio, https://doi.org/10.1142/9789813238053_0012. arXiv: 1706.08749 [hep-ph]
25. R. de Adelhart Toorop, F. Feruglio, C. Hagedorn, Nucl. Phys. B 858, 437-467 (2012). https://doi.org/10.1016/j.nuclphysb.2012. 01.017. arXiv:1112.1340 [hep-ph]

26. J.C. Criado, F. Feruglio, SciPost Phys. 5(5), 042 (2018). https:// doi.org/10.21468/SciPostPhys.5.5.042. arXiv:1807.01125 [hep$\mathrm{ph}]$

27. T. Kobayashi, N. Omoto, Y. Shimizu, K. Takagi, M. Tanimoto, T.H. Tatsuishi, JHEP 11, 196 (2018). https://doi.org/10.1007/ JHEP11(2018)196. arXiv:1808.03012 [hep-ph]

28. H. Okada, M. Tanimoto, Phys. Lett. B 791, 54-61 (2019). https:// doi.org/10.1016/j.physletb.2019.02.028. arXiv:1812.09677 [hep$\mathrm{ph}]$

29. T. Nomura, H. Okada, Phys. Lett. B 797, 134799 (2019). https:// doi.org/10.1016/j.physletb.2019.134799. arXiv:1904.03937 [hep-ph]

30. H. Okada, M. Tanimoto, Eur. Phys. J. C 81(1), 52 (2021). https:// doi.org/10.1140/epjc/s10052-021-08845-y. arXiv:1905.13421 [hep-ph]

31. F.J. de Anda, S.F. King, E. Perdomo, Phys. Rev. D 101(1), 015028 (2020). https://doi.org/10.1103/PhysRevD.101.015028. arXiv: 1812.05620 [hep-ph]

32. P.P. Novichkov, S.T. Petcov, M. Tanimoto, Phys. Lett. B 793, 247-258 (2019). https://doi.org/10.1016/j.physletb.2019.04.043. arXiv:1812.11289 [hep-ph]

33. T. Nomura, H. Okada, Nucl. Phys. B 966, 115372 (2021). https:// doi.org/10.1016/j.nuclphysb.2021.115372. arXiv:1906.03927 [hep-ph]

34. H. Okada, Y. Orikasa, arXiv:1907.13520 [hep-ph]

35. G.J. Ding, S.F. King, X.G. Liu, JHEP 09, 074 (2019). https://doi. org/10.1007/JHEP09(2019)074. arXiv:1907.11714 [hep-ph]

36. T. Nomura, H. Okada, O. Popov, Phys. Lett. B 803, 135294 (2020). https://doi.org/10.1016/j.physletb.2020.135294. arXiv:1908.07457 [hep-ph]

37. T. Kobayashi, Y. Shimizu, K. Takagi, M. Tanimoto, T.H. Tatsuishi, Phys. Rev. D 100(11), 115045 (2019). [Erratum: Phys. Rev. D 101(3), 039904 (2020)]. https://doi.org/10.1103/PhysRevD.100. 115045. arXiv:1909.05139 [hep-ph]

38. T. Asaka, Y. Heo, T.H. Tatsuishi, T. Yoshida, JHEP 01, 144 (2020). https://doi.org/10.1007/JHEP01(2020)144. arXiv:1909.06520 [hep-ph]

39. D. Zhang, Nucl. Phys. B 952, 114935 (2020). https://doi.org/10. 1016/j.nuclphysb.2020.114935. arXiv:1910.07869 [hep-ph]

40. G.J. Ding, S.F. King, X.G. Liu, J.N. Lu, JHEP 12, 030 (2019). https://doi.org/10.1007/JHEP12(2019)030. arXiv:1910.03460 [hep-ph]

41. T. Kobayashi, T. Nomura, T. Shimomura, Phys. Rev. D 102(3), 035019 (2020). https://doi.org/10.1103/PhysRevD.102.035019. arXiv:1912.00637 [hep-ph]

42. T. Nomura, H. Okada, S. Patra, Nucl. Phys. B 967, 115395 (2021). https://doi.org/10.1016/j.nuclphysb.2021.115395. arXiv:1912.00379 [hep-ph]

43. X. Wang, Nucl. Phys. B 957, 115105 (2020). https://doi.org/10. 1016/j.nuclphysb.2020.115105. arXiv:1912.13284 [hep-ph]

44. H. Okada, Y. Shoji, Nucl. Phys. B 961, 115216 (2020). https://doi. org/10.1016/j.nuclphysb.2020.115216. arXiv:2003.13219 [hep$\mathrm{ph}]$

45. H. Okada, M. Tanimoto, arXiv:2005.00775 [hep-ph]

46. M.K. Behera, S. Singirala, S. Mishra, R. Mohanta, arXiv:2009.01806 [hep-ph]

47. M.K. Behera, S. Mishra, S. Singirala, R. Mohanta, arXiv:2007.00545 [hep-ph]

48. T. Nomura, H. Okada, arXiv:2007.04801 [hep-ph]

49. T. Nomura, H. Okada, arXiv:2007.15459 [hep-ph]

50. T. Asaka, Y. Heo, T. Yoshida, Phys. Lett. B 811, 135956 (2020). https://doi.org/10.1016/j.physletb.2020.135956. arXiv:2009.12120 [hep-ph] 
51. H. Okada, M. Tanimoto, Phys. Rev. D 103(1), 015005 (2021). https://doi.org/10.1103/PhysRevD.103.015005. arXiv:2009.14242 [hep-ph]

52. K.I. Nagao, H. Okada, arXiv:2010.03348 [hep-ph]

53. H. Okada, M. Tanimoto, JHEP 03, 010 (2021). https://doi.org/10. 1007/JHEP03(2021)010. arXiv:2012.01688 [hep-ph]

54. C.Y. Yao, J.N. Lu, G.J. Ding, JHEP 05, 102 (2021). https://doi. org/10.1007/JHEP05(2021)102. arXiv:2012.13390 [hep-ph]

55. P. Chen, G.J. Ding, S.F. King, JHEP 04, 239 (2021). https://doi. org/10.1007/JHEP04(2021)239. arXiv:2101.12724 [hep-ph]

56. T. Kobayashi, T. Shimomura, M. Tanimoto, arXiv:2102.10425 [hep-ph]

57. M. Kashav, S. Verma, arXiv:2103.07207 [hep-ph]

58. H. Okada, Y. Shimizu, M. Tanimoto, T. Yoshida, arXiv:2105.14292 [hep-ph]

59. T. Kobayashi, K. Tanaka, T.H. Tatsuishi, Phys. Rev. D 98(1), 016004 (2018). https://doi.org/10.1103/PhysRevD.98.016004. arXiv:1803.10391 [hep-ph]

60. T. Kobayashi, Y. Shimizu, K. Takagi, M. Tanimoto, T.H. Tatsuishi, H. Uchida, Phys. Lett. B 794, 114-121 (2019). https://doi.org/10. 1016/j.physletb.2019.05.034. arXiv:1812.11072 [hep-ph]

61. T. Kobayashi, Y. Shimizu, K. Takagi, M. Tanimoto, T.H. Tatsuishi, PTEP 2020(5), 053B05 (2020). https://doi.org/10.1093/ ptep/ptaa055. arXiv:1906.10341 [hep-ph]

62. H. Okada, Y. Orikasa, Phys. Rev. D 100(11), 115037 (2019). $\quad$ https://doi.org/10.1103/PhysRevD.100.115037. arXiv:1907.04716 [hep-ph]

63. S. Mishra, arXiv:2008.02095 [hep-ph]

64. X. Du, F. Wang, JHEP 02, 221 (2021). https://doi.org/10.1007/ JHEP02(2021)221. arXiv:2012.01397 [hep-ph]

65. J.T. Penedo, S.T. Petcov, Nucl. Phys. B 939, 292-307 (2019). https://doi.org/10.1016/j.nuclphysb.2018.12.016. arXiv: 1806.11040 [hep-ph]

66. P.P. Novichkov, J.T. Penedo, S.T. Petcov, A.V. Titov, JHEP 04, 005 (2019). https://doi.org/10.1007/JHEP04(2019)005. arXiv:1811.04933 [hep-ph]

67. T. Kobayashi, Y. Shimizu, K. Takagi, M. Tanimoto, T.H. Tatsuishi, JHEP 02, 097 (2020). https://doi.org/10.1007/JHEP02(2020)097. arXiv:1907.09141 [hep-ph]

68. S.F. King, Y.L. Zhou, Phys. Rev. D 101(1), 015001 (2020). https:// doi.org/10.1103/PhysRevD.101.015001. arXiv:1908.02770 [hep-ph]

69. H. Okada, Y. Orikasa, arXiv:1908.08409 [hep-ph]

70. J.C. Criado, F. Feruglio, S.J.D. King, JHEP 02, 001 (2020). https:// doi.org/10.1007/JHEP02(2020)001. arXiv:1908.11867 [hep-ph]

71. X. Wang, S. Zhou, JHEP 05, 017 (2020). https://doi.org/10.1007/ JHEP05(2020)017. arXiv:1910.09473 [hep-ph]

72. Y. Zhao, H.H. Zhang, JHEP 03, 002 (2021). https://doi.org/10. 1007/JHEP03(2021)002. arXiv:2101.02266 [hep-ph]

73. S.F. King, Y.L. Zhou, JHEP 04, 291 (2021). https://doi.org/10. 1007/JHEP04(2021)291. arXiv:2103.02633 [hep-ph]

74. G.J. Ding, S.F. King, C.Y. Yao, arXiv:2103.16311 [hep-ph]

75. X. Zhang, S. Zhou, arXiv:2106.03433 [hep-ph]

76. B.-Y. Qu, X.-G. Liu, P.-T. Chen, G.-J. Ding, arXiv:2106.11659 [hep-ph]

77. P.P. Novichkov, J.T. Penedo, S.T. Petcov, A.V. Titov, JHEP 04, 174 (2019). https://doi.org/10.1007/JHEP04(2019)174. arXiv:1812.02158 [hep-ph]

78. G.J. Ding, S.F. King, X.G. Liu, Phys. Rev. D 100(11), 115005 (2019). https://doi.org/10.1103/PhysRevD.100.115005. arXiv: 1903.12588 [hep-ph]

79. X. Wang, B. Yu, S. Zhou, Phys. Rev. D 103(7), 076005 (2021). https://doi.org/10.1103/PhysRevD.103.076005. arXiv:2010.10159 [hep-ph]
80. C.Y. Yao, X.G. Liu, G.J. Ding, Phys. Rev. D 103(9), 095013 (2021). https://doi.org/10.1103/PhysRevD.103.095013. arXiv:2011.03501 [hep-ph]

81. X. Wang, S. Zhou, arXiv:2102.04358 [hep-ph]

82. A. Baur, H.P. Nilles, A. Trautner, P.K.S. Vaudrevange, Phys. Lett. B 795, 7-14 (2019). https://doi.org/10.1016/j.physletb.2019.03. 066. arXiv:1901.03251 [hep-th]

83. I. de Medeiros Varzielas, S.F. King, Y.L. Zhou, Phys. Rev. D 101(5), 055033 (2020). https://doi.org/10.1103/PhysRevD.101. 055033. arXiv:1906.02208 [hep-ph]

84. X.G. Liu, G.J. Ding, JHEP 08, 134 (2019). https://doi.org/10. 1007/JHEP08(2019)134. arXiv:1907.01488 [hep-ph]

85. P. Chen, G.J. Ding, J.N. Lu, J.W.F. Valle, Phys. Rev. D 102(9), 095014 (2020). https://doi.org/10.1103/PhysRevD.102.095014. arXiv:2003.02734 [hep-ph]

86. P.P. Novichkov, J.T. Penedo, S.T. Petcov, Nucl. Phys. B 963, 115301 (2021). https://doi.org/10.1016/j.nuclphysb.2020. 115301. arXiv:2006.03058 [hep-ph]

87. X.G. Liu, C.Y. Yao, G.J. Ding, Phys. Rev. D 103(5), 056013 (2021). https://doi.org/10.1103/PhysRevD.103.056013. arXiv:2006.10722 [hep-ph]

88. S. Kikuchi, T. Kobayashi, H. Otsuka, S. Takada, H. Uchida, JHEP 11, 101 (2020). https://doi.org/10.1007/JHEP11(2020)101. arXiv:2007.06188 [hep-th]

89. Y. Almumin, M.C. Chen, V. Knapp-Pérez, S. Ramos-Sánchez, M. Ratz, S. Shukla, JHEP 05, 078 (2021). https://doi.org/10.1007/ JHEP05(2021)078. arXiv:2102.11286 [hep-th]

90. G.J. Ding, F. Feruglio, X.G. Liu, SciPost Phys. 10, 133 (2021). $\quad$ https://doi.org/10.21468/SciPostPhys.10.6.133. arXiv:2102.06716 [hep-ph]

91. F. Feruglio, V. Gherardi, A. Romanino, A. Titov, JHEP 05, 242 (2021). https://doi.org/10.1007/JHEP05(2021)242. arXiv:2101.08718 [hep-ph]

92. S. Kikuchi, T. Kobayashi, H. Uchida, arXiv:2101.00826 [hep-th]

93. P.P. Novichkov, J.T. Penedo, S.T. Petcov, JHEP 04, 206 (2021). https://doi.org/10.1007/JHEP04(2021)206. arXiv:2102.07488 [hep-ph]

94. G. Altarelli, F. Feruglio, Rev. Mod. Phys. 82, 2701-2729 (2010). https://doi.org/10.1103/RevModPhys.82.2701. arXiv:1002.0211 [hep-ph]

95. H. Ishimori, T. Kobayashi, H. Ohki, Y. Shimizu, H. Okada, M. Tanimoto, Prog. Theor. Phys. Suppl. 183, 1-163 (2010). https:// doi.org/10.1143/PTPS.183.1. arXiv:1003.3552 [hep-th]

96. H. Ishimori, T. Kobayashi, H. Ohki, H. Okada, Y. Shimizu, M. Tanimoto, Lect. Notes Phys. 858, 1-227 (2012). https://doi.org/ 10.1007/978-3-642-30805-5

97. D. Hernandez, A.Y. Smirnov, Phys. Rev. D 86, 053014 (2012). https://doi.org/10.1103/PhysRevD.86.053014. arXiv:1204.0445 [hep-ph]

98. S.F. King, C. Luhn, Rep. Prog. Phys. 76, 056201 (2013). https:// doi.org/10.1088/0034-4885/76/5/056201. arXiv:1301.1340 [hep-ph]

99. S.F. King, A. Merle, S. Morisi, Y. Shimizu, M. Tanimoto, New J. Phys. 16, 045018 (2014). https://doi.org/10.1088/1367-2630/16/ 4/045018. arXiv:1402.4271 [hep-ph]

100. S.F. King, Prog. Part. Nucl. Phys. 94, 217-256 (2017). https://doi. org/10.1016/j.ppnp.2017.01.003. arXiv:1701.04413 [hep-ph]

101. S.T. Petcov, Eur. Phys. J. C 78(9), 709 (2018). https://doi.org/10. 1140/epjc/s10052-018-6158-5. arXiv:1711.10806 [hep-ph]

102. A. Baur, H.P. Nilles, A. Trautner, P.K.S. Vaudrevange, Nucl. Phys. B 947, 114737 (2019). https://doi.org/10.1016/j.nuclphysb.2019. 114737. arXiv:1908.00805 [hep-th]

103. T. Kobayashi, Y. Shimizu, K. Takagi, M. Tanimoto, T.H. Tatsuishi, H. Uchida, Phys. Rev. D 101(5) (2020). https://doi.org/10.1103/ PhysRevD.101.055046. arXiv:1910.11553 [hep-ph] 
104. P.P. Novichkov, J.T. Penedo, S.T. Petcov, A.V. Titov, JHEP 07, 165 (2019). https://doi.org/10.1007/JHEP07(2019)165. arXiv:1905.11970 [hep-ph]

105. M. Tanimoto, K. Yamamoto, arXiv:2106.10919 [hep-ph]

106. M.C. Chen, S. Ramos-Sánchez, M. Ratz, Phys. Lett. B 801, 135153 (2020). https://doi.org/10.1016/j.physletb.2019.135153. arXiv:1909.06910 [hep-ph]

107. I. de Medeiros Varzielas, M. Levy, Y.L. Zhou, JHEP 11, 085 (2020). https://doi.org/10.1007/JHEP11(2020)085. arXiv:2008.05329 [hep-ph]

108. K. Ishiguro, T. Kobayashi, H. Otsuka, JHEP 03, 161 (2021). https://doi.org/10.1007/JHEP03(2021)161. arXiv:2011.09154 [hep-ph]

109. I. Esteban, M.C. Gonzalez-Garcia, M. Maltoni, T. Schwetz, A. Zhou, JHEP 09, 178 (2020). https://doi.org/10.1007/ JHEP09(2020)178. arXiv:2007.14792 [hep-ph]
110. A. Gando et al. [KamLAND-Zen], Phys. Rev. Lett. 117(8), 082503 (2016). https://doi.org/10.1103/PhysRevLett.117. 082503. arXiv:1605.02889 [hep-ex]

111. Z. Maki, M. Nakagawa, S. Sakata, Prog. Theor. Phys. 28, 870-880 (1962). https://doi.org/10.1143/PTP.28.870

112. P.A. Zyla et al. (Particle Data Group), Prog. Theor. Exp. Phys. 2020, 083 C01 (2020)

113. G. Aad et al. [ATLAS], Eur. Phys. J. C 81(4), 313 (2021). https:// doi.org/10.1140/epjc/s10052-021-09009-8. arXiv:2010.02098 [hep-ex]

114. G. Aad et al. [ATLAS], JHEP 06, 179 (2021). arXiv:2101.11582 [hep-ex]

115. A.M. Sirunyan et al. [CMS], Phys. Lett. B 819, 136446 (2021). arXiv:2012.04178 [hep-ex] 